\title{
Electroweak corrections to diphoton plus jets
}

\author{
Mauro Chiesa, ${ }^{a}$ Nicolas Greiner, ${ }^{b}$ Marek Schönherr ${ }^{b}$ and Francesco Tramontano $^{c}$ \\ ${ }^{a}$ Institut für Theoretische Physik und Astrophysik, Julius-Maximilians-Universität Würzburg, \\ Emil-Hilb-Weg 22, D-97074 Würzburg, Germany \\ ${ }^{b}$ Physik Institut, Universität Zürich, \\ Winterthurerstr. 190, 8057 Zürich, Switzerland \\ ${ }^{c}$ Dipartimento di Fisica, Università di Napoli "Federico II" and INFN, sezione di Napoli, \\ via Cintia, 80126 Napoli, Italy \\ E-mail: mauro.chiesa@physik.uni-wuerzburg.de, greiner@physik.uzh.ch, \\ marek.schoenherr@physik.uzh.ch, francesco.tramontano@cern.ch
}

ABSTRACT: We calculate the next-to-leading order electroweak corrections to the production of a photon pair in association with zero, one and two jets at the LHC. We use GoSAM and SHERPA to obtain the results in a fully automated way. For a typical set of fiducial cuts the electroweak corrections lead to a modification of the total cross section of up to $3 \%$, depending on the jet multiplicity. We find substantial contributions in differential distributions, leading to tens of per cent corrections for phase space regions within the reach of the LHC. Furthermore we investigate the importance of photon induced processes as well as subleading contributions. Photon induced processes are found to be negligible, subleading contributions can have a sizeable impact however they can be removed by appropriate phase space cuts.

KeYwords: NLO Computations

ARXIV EPRINT: 1706.09022 


\section{Contents}

1 Introduction 1

2 Calculational setup $\quad 2$

3 Numerical results 3

4 Conclusions $\quad 12$

\section{Introduction}

The production of a pair of photons is an interesting and important process at the LHC. It played an essential role in the discovery of the Higgs boson [1, 2] and in further refined measurements of fiducial cross sections and differential observables [3-7]. Due to its clean experimental signal, diphoton production in hadron collisions offers a testing ground for perturbative quantum chromodynamics and has been widely studied at the LHC [8-12]. This process is also an important search channel for new physics (see [13-20] for recent experimental searches). Diphoton signals can occur in models with extra spatial components or in cascade decays of heavy new particles. Diphoton signals in association with jets and missing energy occur in gauge mediated SUSY scenarios.

Diphoton searches at the LHC are typically carried out fully inclusively in the number of jets. In fact, due to the hadronic environment there is a large probability that the photon pair will be accompanied by one or more high energetic jets. The two jet process is particularly interesting since it allows to asses the Vector-Boson-Fusion (VBF) production channel.

The next-to-leading (NLO) QCD corrections to diphoton production at hadron colliders have been computed in refs. [21, 22] and they have been implemented in the fixed order codes Diphox [23], 2gammaMc [24], MCFM [25], VBF@NLO [26]. Theoretical predictions for $p p \rightarrow \gamma \gamma$ at QCD NLO matched with parton shower can be found in ref. [27], while in ref. [28] diphoton production is studied in the context of matrix element-parton shower merging. Soft gluon resummation for $p p \rightarrow \gamma \gamma$ has been performed in [29-31]. The diphoton process is known at NNLO in QCD [32, 33], at NLO in the electroweak coupling constant [34]. The diphoton plus one and two jet processes are known at next to leading order in QCD [35-39].

In this paper we calculate the next-to-leading order electroweak corrections to the production of a pair of photons in association with up to two jets. More precisely we calculate the electroweak corrections to the leading contribution that are of the order $\mathcal{O}\left(\alpha^{2} \alpha_{s}^{i}\right)$ with $i=0,1,2$, where $i$ denotes the number of jets. This calculation provides 
important results that are useful for measurements of a diphoton system in association with jets. They are particularly useful for background studies for Higgs production as well as in the context of boosted searches where one expects sizeable effects from electroweak corrections. In addition we asses the impact of photon induced processes as well the impact of subleading electroweak contributions. The paper is organized as follows. In section 2 we describe the calculational setup before we discuss the results and the phenomenology of these processes in section 3. Finally we conclude in section 4 .

\section{Calculational setup}

The calculation has been performed by combining the functionalities of the GoSAM [40, 41] and SHERPA [42] programs. The GOSAM package generates the code for the numerical evaluation of the one loop scattering amplitudes starting from the Feynman diagrams, generated with QGRAF [43], and writing them as $d$-dimensional integrand over the loop momentum, making use of FORM $[44,45]$ and SPINNEY [46] to perform the needed algebraic manipulations. For the integrand reduction of the diagrams we use the NiNJA library [47], an implementation of the technique of integrand reduction via Laurent expansion $[48,49]$. Alternatively one can choose other reduction strategies such as OPP reduction method [50-52] which is implemented in $d$ dimensions in SAMURAI [53], or methods based on tensor integral reduction as implemented in GoLEM95 [54-57]. We have used OnELOOP [58] to evaluate the scalar integrals.

One loop electroweak renormalization is performed in the on-shell scheme (see [60] and references therein) within the complex-mass-scheme framework [61-63]. Since for the processes under consideration the powers of $\alpha$ only come from $\gamma f \bar{f}$ vertices, we use the $\alpha(0)$ input parameter scheme. All fermion masses, except for the top, are set to zero. This choice of input parameters leads to mass singularities both in the electric charge and in the photon wave function renormalization counterterms $\left(\delta Z_{e}\right.$ and $\left.\delta Z_{A A}\right)$, respectively, however these counterterms only appear in the combination $\delta Z_{e}+\frac{1}{2} \delta Z_{A A}$ where the mass singularities cancel. Counterterm functions are first computed in conventional dimension regularization (CDR) and then converted in dimensional reduction (DRED) as detailed in [59], to be used within the GoSAM framework.

The present paper is the first application of a new module in GoSAM for the fully automated generation of the electroweak renormalization counter terms.

SHERPA, on the other hand, is used for providing all tree-level matrix elements, infrared subtraction, process management and phase-space integration of all the contributions to the cross sections considered here [64]. The infrared subtraction is carried out in the QED generalisation of the Catani-Seymour scheme [65-71] and includes the initial state collinear factorisation counter terms. The two programs are interfaced through a dedicated interface based on the Binoth Les Houches Accord [72, 73]. We have cross-checked the treelevel matrix elements of GOSAM with SHERPA and the renormalized pole terms of GOSAM against the infrared pole terms of SHERPA. This has been done for several phase space points spanning multiple kinematic regimes and we have found excellent agreement. 


\section{Numerical results}

In this section we present numerical results for the next-to-leading order electroweak corrections in the production of (at least) two isolated photons, both inclusively and in association with at least one or two jets, pp $\rightarrow \gamma \gamma+0,1,2$ jets at the LHC at a centre-of-mass energy of $13 \mathrm{TeV}$. Our calculation is performed in the Standard Model using the complex-mass scheme with the following input parameters

$$
\begin{aligned}
\alpha(0) & =1 / 137.03599976 & & \\
m_{W} & =80.385 \mathrm{GeV} & \Gamma_{W} & =2.085 \mathrm{GeV} \\
m_{Z} & =91.1876 \mathrm{GeV} & \Gamma_{Z} & =2.4952 \mathrm{GeV} \\
m_{t} & =171.2 \mathrm{GeV} & \Gamma_{t} & =0 .
\end{aligned}
$$

The width of the top quark can be safely neglected as there are no diagrams containing it as an $s$-channel resonance which can potentially go on-shell. At the same time there are no diagrams containing the Higgs boson due to the absence of $W, Z$ or top quark propagators at LO. All other parton masses and widths are set to zero, i.e. we are working in the five-flavour scheme.

Isolated photons are defined with the help of the smooth cone isolation criterion [76] which limits the maximally allowed hadronic activity in a cone of size $R_{\gamma}$ around a photon to

$$
E_{\mathrm{had}, \max }\left(r_{\gamma}\right)=\epsilon p_{T}^{\gamma}\left(\frac{1-\cos r_{\gamma}}{1-\cos R_{\gamma}}\right)^{n}
$$

where $r_{\gamma}$ denotes the angular separation between the photon and the parton. The free parameters $R_{\gamma}, \epsilon$, and $n$ are set to

$$
R_{\gamma}=0.4, \quad \epsilon=0.05, \quad n=1 .
$$

Of the thus found photon candidates we require at least two to lie in the fiducial volume given by

$$
p_{T_{\gamma_{1}}}>40 \mathrm{GeV}, \quad p_{T_{\gamma_{2}}}>30 \mathrm{GeV}, \quad\left|\eta_{\gamma}\right|<2.37 .
$$

It is worth noting that at NLO EW it is possible to find more than two isolated photons, in which case any pair is allowed to fulfill these selection criteria. Of those photons, the one with largest transverse momentum is refered to as $\gamma_{1}$ in the following, while the one with second largest transverse momentum is refered to as $\gamma_{2}$. Any additional identified photons are kept as such. All identified photons are further required to be pairwise separated by $\Delta R\left(\gamma_{i}, \gamma_{j}\right)>0.4$, otherwise the event is discarded.

On the other hand, photons that are either not isolated according to eq. (3.1) or do not fulfill the cuts of eq. (3.3) are passed to the jet algorithm, along with all quarks and gluons of the event. Jet candidates are then found using the anti- $k_{t}$ clustering algorithm [78] with a cone size of $R=0.4$ provided by the FASTJET package $[79,80]$. The candidates are further required to have

$$
p_{\mathrm{T}_{j}}>30 \mathrm{GeV}, \quad\left|y_{j}\right|<4.4 .
$$

At this point we want to stress that the best procedure would be a democratic clustering [77], where photons and partons are treated on the same footing and clustered by an 


\begin{tabular}{|l|c|c|c|}
\hline & $p p \rightarrow \gamma \gamma$ & $p p \rightarrow \gamma \gamma+j$ & $p p \rightarrow \gamma \gamma+j j$ \\
\hline \hline$\sigma_{\mathrm{LO}}[\mathrm{pb}]$ & 5.398 & 9.597 & 2.073 \\
\hline$\sigma_{\mathrm{NLO} E W}[\mathrm{pb}]$ & 5.449 & 9.587 & 2.009 \\
\hline$\delta_{\mathrm{EW}}[\%]$ & 0.9 & -0.1 & -3.1 \\
\hline
\end{tabular}

Table 1. Total cross sections at LO and NLO for $\gamma \gamma+0,1,2$ jets production at $13 \mathrm{TeV}$ at the LHC.

infrared safe jet algorithm. However this necessitates the inclusion of additional components in the NLO calculation. Indeed, one can have configurations where a hard photon is accompanied by a soft gluon forming a jet. Such a configuration would lead to a QCD singularity which requires the virtual QCD corrections to a different underlying Born, where the gluon is replaced by a photon, to cancel this singularity. Thus if a photon and a gluon are clustered to a jet the gluon is required to carry a non-vanishing fraction of the momentum of the jet to avoid the occurrence of QCD infrared singularities. The difference to the scheme used in this paper is estimated to be very small [69]. To ensure infrared safety wrt. possible soft gluons a photon must not carry a transverse energy fraction of more than $z_{\text {thr }}=0.5$ within a jet, if the jet comprises a photon and a gluon. This constitutes a less invasive version of the regularisation applied in [69, 70]. A further requirement of $\Delta R\left(\gamma_{i}, j_{j}\right)>0.4$ removes the overlap between identified photons and jets.

The renormalization and factorization scales are chosen to be equal and set to

$$
\mu_{R, F}=\frac{1}{2} \sqrt{m_{\gamma \gamma}^{2}+\hat{H}_{\mathrm{T}}^{2}} \quad \text { with } \quad \hat{H}_{\mathrm{T}}=\sum_{i} p_{\mathrm{T}, i}
$$

wherein the sum runs over all partons but the identified photons [37]. As our main focus in this paper is on the size of the corrections, i.e. the ratio with respect to the leading order and as we do not expect significant deviations from a variation, we do not vary the scale but leave it fixed at the central value.

The invariant diphoton mass is formed using the two leading identified photons defined above. We use the CT14nlo PDF [74] set, interfaced through LHAPDF6 [75], with its accompanying $\alpha_{s}$-parametrisation with $\alpha_{s}\left(m_{Z}\right)=0.118$ throughout. If not stated otherwise we neglect all photon initiated processes. Due to the smallness of the photon PDF and the fact that they enter only at NLO EW, their impact is estimated to be negligible. We will quantify this statement later in this section.

The total cross sections for the three different processes are listed in table 1 . The first interesting point is the fact that, at leading order and next-to-leading order electroweak, the fiducial cross section for the 1-jet process is almost a factor of two bigger than the 0 -jet process. This is due to the quark-gluon channel opening up for the 1-jet case whereas the 0 -jet process is mediated only by $q \bar{q}$ initial states. For the 2 -jet process there is then also the gluon-gluon initial state possible. This leads to the fact that the 2 -jet process is only roughly a factor of two smaller than the 0 -jet process although it is suppressed by two additional powers of $\alpha_{s}$. This is remedied by the inclusion of higher order QCD correction [32], but is beyond the scope of this paper. The NLO EW corrections to the total 


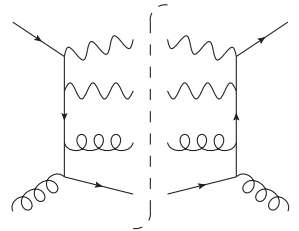

$\mathcal{O}\left(\alpha_{s}^{2} \alpha^{2}\right)$

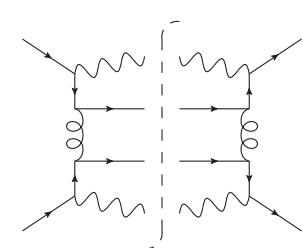

$\mathcal{O}\left(\alpha_{s}^{2} \alpha^{2}\right)$

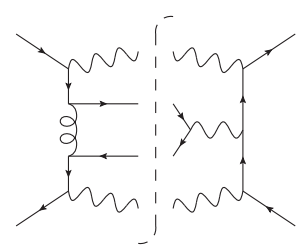

$\mathcal{O}\left(\alpha_{s} \alpha^{3}\right)$

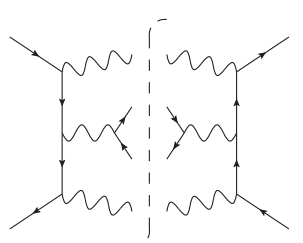

$\mathcal{O}\left(\alpha^{4}\right)$

Figure 1. Illustrative leading and sub-leading tree-level diagrams for $\gamma \gamma+j j$ production.
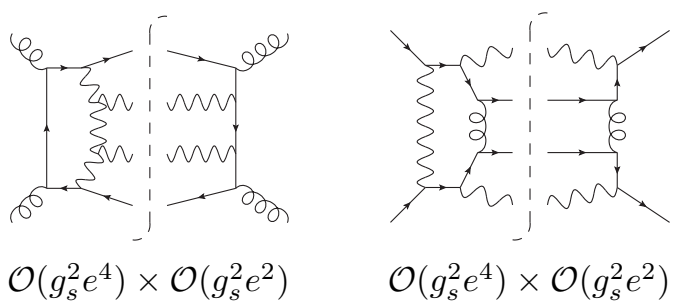

$\mathcal{O}\left(g_{s}^{2} e^{4}\right) \times \mathcal{O}\left(g_{s}^{2} e^{2}\right)$

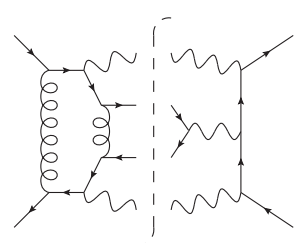

$\mathcal{O}\left(g_{s}^{4} e^{2}\right) \times \mathcal{O}\left(e^{4}\right)$

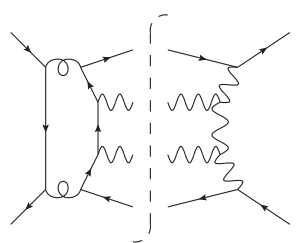

$\mathcal{O}\left(g_{s}^{4} e^{2}\right) \times \mathcal{O}\left(e^{4}\right)$

Figure 2. Representative virtual corrections at $\mathcal{O}\left(\alpha_{s}^{2} \alpha^{3}\right)$ for $\gamma \gamma+j j$ production.

cross section are tiny, for the 0-jet process they are positive and lead to an enhancement of one per cent. Increasing the jet multiplicity reduces the EW corrections and they become negative, for the 1 -jet process the cross section is essentially unchanged, for the 2 -jet process we find a reduction of three per cent.

We now turn to the discussion of a selection of the differential distributions where one expects to see a larger impact of the EW corrections than for the total cross sections, predominantly at large $p_{\mathrm{T}}$. For the 2-jet process we additionally include two different subleading contributions at tree-level: 'NLO $\mathrm{EW}^{*}$ ' includes also $\mathcal{O}\left(\alpha_{s} \alpha^{3}\right)$ contributions, and 'NLO EW ${ }^{* *}$ ' includes both the $\mathcal{O}\left(\alpha_{s} \alpha^{3}\right)$ and $\mathcal{O}\left(\alpha^{4}\right)$ terms.

The subleading Born contributions at $\mathcal{O}\left(\alpha_{s} \alpha^{3}\right)$ arise from interferences of "QCD" production diagrams at $\mathcal{O}\left(g_{s}^{2} e^{2}\right)$ and "EW" production diagrams at $\mathcal{O}\left(e^{4}\right)$, as detailed in figure 1. As such, they are not a proiri positive definite. Similar considerations arise for the virtual corrections at $\mathcal{O}\left(\alpha_{s}^{2} \alpha^{3}\right)$, which include pieces that are both either insertions of electroweak loops into tree-level processes of $\mathcal{O}\left(\alpha_{s}^{2} \alpha^{2}\right)$ or insertions of QCD loops into interference contributions of $\mathcal{O}\left(\alpha_{s} \alpha^{3}\right)$, cf. figure 2 .

The contributions at $\mathcal{O}\left(\alpha^{4}\right)$ are pure absolute squares of diagrams at $\mathcal{O}\left(e^{4}\right)$ and we will denote them "sub-subleading" in the following. Their contribution is to be considered with care as a significant, if not dominant, fraction of the contribution arises from near resonant $\gamma \gamma V^{*}(V=W, Z)$ production with subsequent hadronic decays of the vector boson. At this point, it is a matter of definition of the analysis strategy whether these processes are to be included as signal or whether they are treated as background. In the latter case they, of course, would not be considered to be contributing to the $\gamma \gamma+2$ jets signal. Similarly, they could be effectively removed by vetoing events with dijet invariant masses in a generous window around the nominal electroweak vector boson masses. 


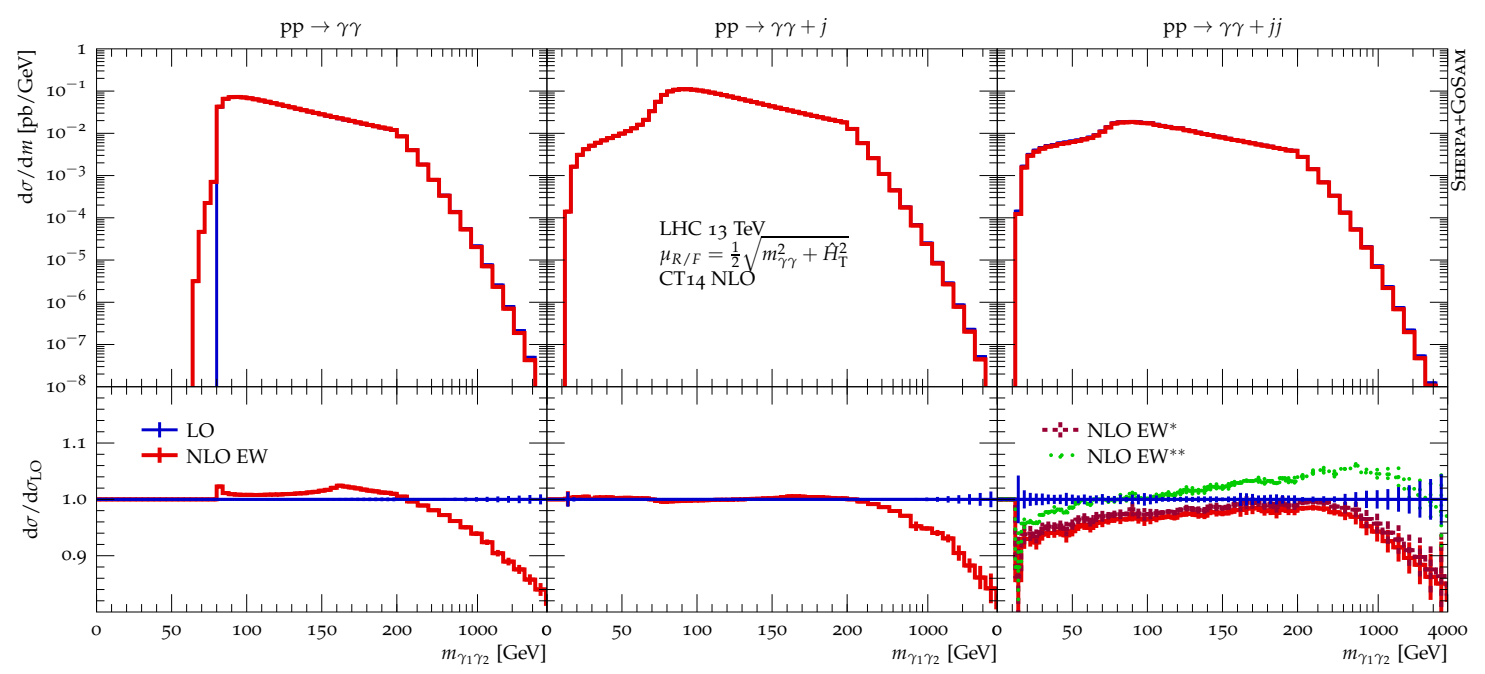

Figure 3. The invariant mass of the leading and subleading isolated photons in $\gamma \gamma+0,1,2$ jets production at $13 \mathrm{TeV}$ at the LHC. Note that above $200 \mathrm{GeV}$ the $x$-axis is given as a logarithmic scale.

We start our discussion of the differential distributions with the invariant mass distribution of the two leading photons. This is shown in figure 3 . The 0 -jet process is a somewhat special case as at LO the invariant mass is strictly determined by the transverse momentum of the two photons and the back-to-back kinematic of the leading order configuration. This back-to-back requirement is then relaxed by the real emission, but it requires an additional partonic jet at leading order to allow for small invariant masses of the diphoton system. For the 0 -jet process one obtains a small positive correction for low invariant masses with a small peak at around $80 \mathrm{GeV}$ and at $2 \cdot m_{W}$. It is these peaks that lead to the overall positive correction to the total cross section. Looking more closely at the ingredients that contribute to the NLO result we see that the peak at $2 m_{W}$ is generated by the virtual corrections. More precisely it originates from the class of diagrams that we illustrated in the left sample diagram of figure 2 if one just removes the gluons and crosses the quarks into the initial state. The lower peak is caused by the real emission contribution and is therefore a threshold effect, as at leading order the minimal value for the invariant mass is $80 \mathrm{GeV}$. For large values of the invariant mass one obtains the typical decrease the cross section due to the negative Sudakov logarithms of the virtual contributions. For invariant masses of the order of $1 \mathrm{TeV}$ one obtains a ten per cent correction. For the 1-jet process the EW corrections are essentially zero for small invariant masses and only from $\sim 200 \mathrm{GeV}$ onwards one observes the typical decrease which however is irrelevant for the total cross section. For the 2-jet process we obtain negative corrections of the order of $5-10 \%$ already for low invariant masses below the electroweak scale. They reach their minimum for intermediate scales of up to a few hundred $\mathrm{GeV}$ before one finds the decrease of the cross section caused by the Sudakov logarithms. It is worth mentioning that the subleading Born contribution do not play a significant role, but they are superseded by the sub-subleading Born contributions. They lead to an overall positive shift which becomes 


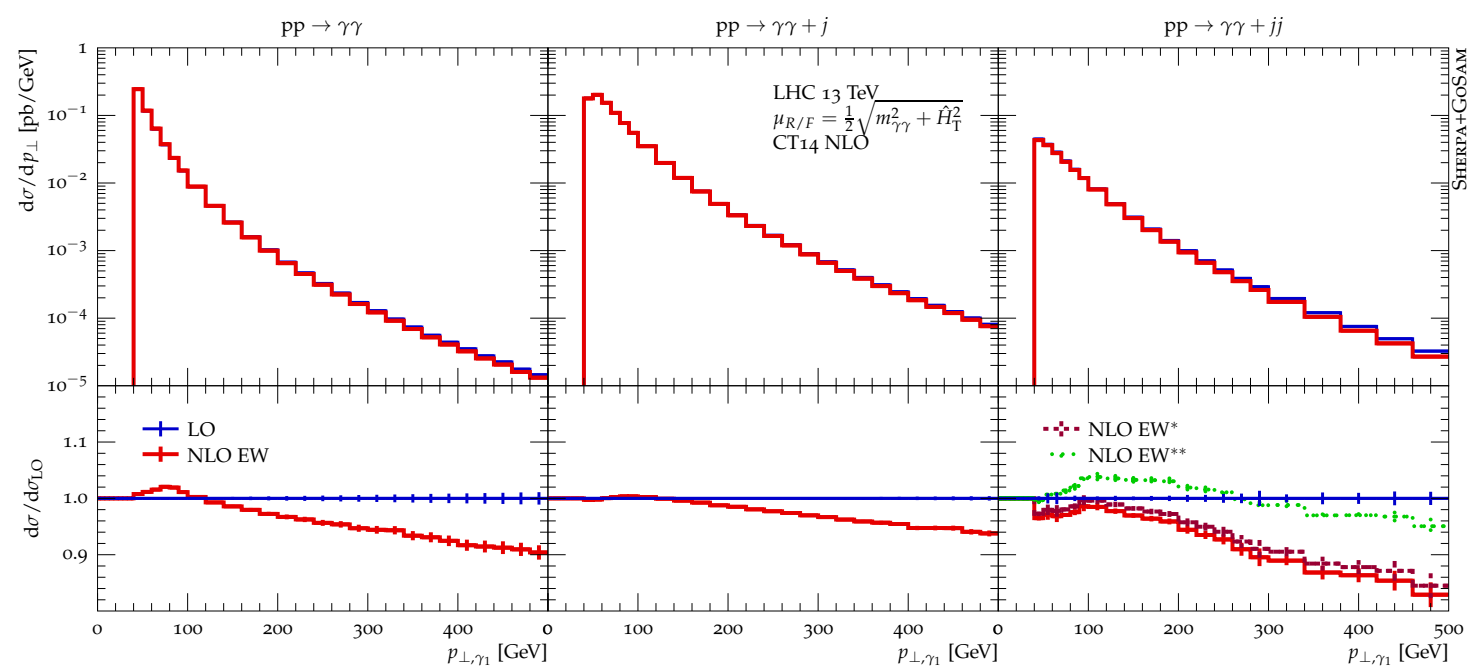

Figure 4. The transverse momentum of the leading isolated photon in $\gamma \gamma+0,1,2$ jets production at $13 \mathrm{TeV}$ at the LHC.

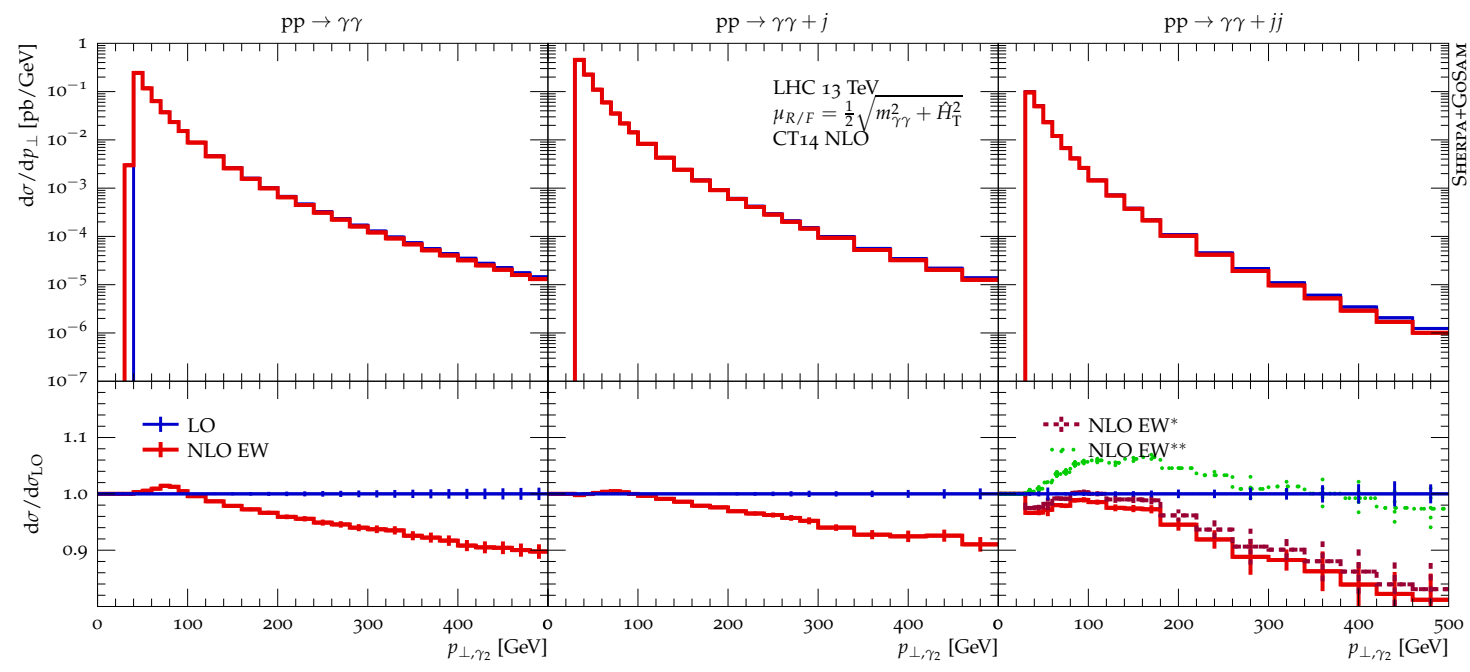

Figure 5. The transverse momentum of the subleading isolated photon in $\gamma \gamma+0,1,2$ jets production at $13 \mathrm{TeV}$ at the LHC.

more enhanced for high invariant masses. It would be interesting to investigate how their effect would change at NLO EW which is however beyond the scope of this paper.

A similar pattern can be seen for the transverse momenta of the leading and subleading photon which are shown in figure 4 and figure 5 respectively, the electroweak corrections affect both distributions in the same way. For the 0-jet process one obtains a small positive correction for low transverse momenta up to about $100 \mathrm{GeV}$. This peak is related to the corresponding ones described above for the diphoton invariant mass distribution. It is followed by a decrease that leads a $10 \%$ correction for transverse momenta of $500 \mathrm{GeV}$. For the 1-jet process this effect appears to be a bit milder. For the 2-jet process we observe a negative correction for small $p_{T}$ that vanishes around the weak scale and then becomes 


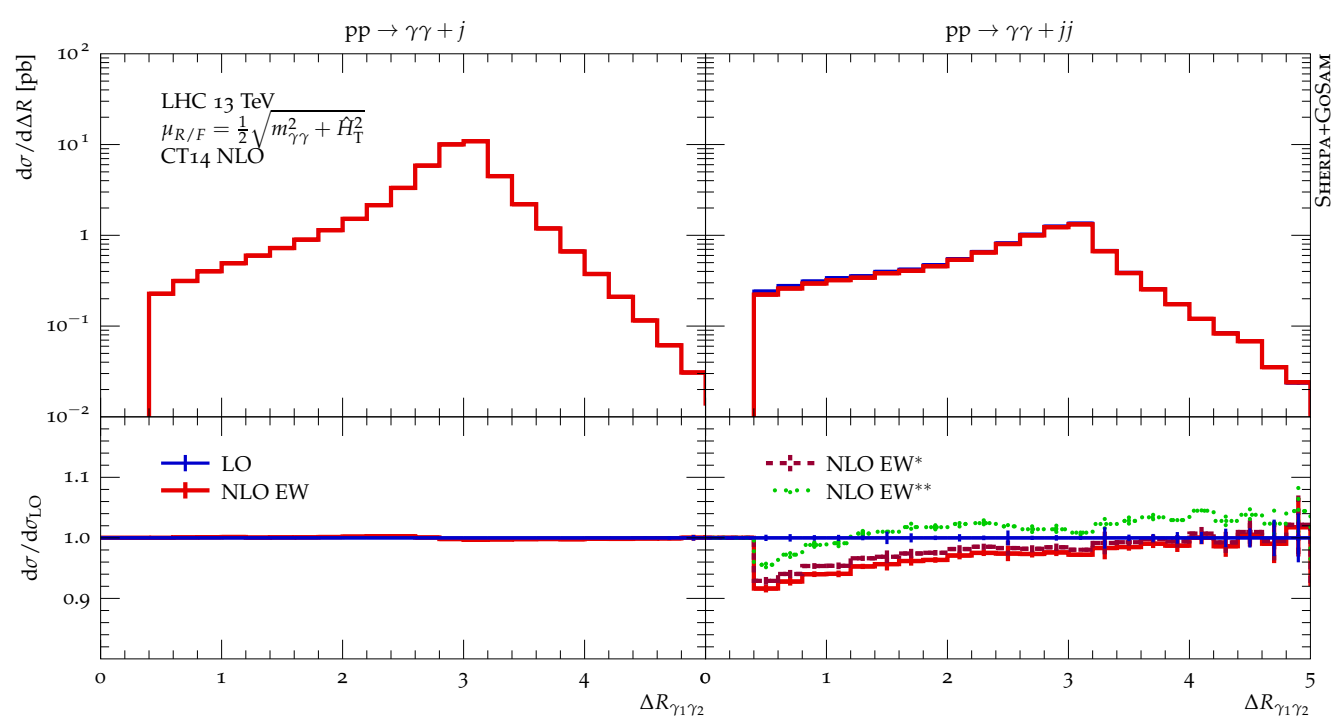

Figure 6. Angular separation of the leading and subleading isolated photon system in $\gamma \gamma+1,2$ jets production at $13 \mathrm{TeV}$ at the LHC.

larger again in the Sudakov regime leading to a $\sim 20 \%$ correction for large transverse momenta of around $500 \mathrm{GeV}$. Also here we see that the subleading born leads to a small correction and the much bigger effect, in particular for the subleading photon, comes from the sub-subleading Born contribution. In the tail it leads to an upward shift of almost $20 \%$ that essentially nullifies the electroweak corrections to the leading Born. But as mentioned above this has to be interpreted with care as one then can also expect the electroweak corrections to the sub-subleading Born to be of non-negligible size.

The angular separation between the two leading photons is shown in figure 6 . We do not plot the 0-jet contribution as deviations from the back-to-back kinematic are only of leading order accuracy in that case. For the 1-jet process we find that the corrections are basically zero. This is not surprising when having in mind that for the total cross section the electroweak correction were negligible and the corrections are only relevant in the high energy tails. As the angular distributions mix these regions more or less evenly one cannot expect significant deviations. For the 2-jet process we find substantial deviations of the order of $10 \%$ for small values of the angular separation and the corrections become more and more negligible when increasing the angular separation.

Following this line of arguments it is not surprising that the azimuthal separation which is shown in figure 7 , follows the same pattern as what we have discussed for the angular separation. Also here we find that the corrections in the 1-jet process are negligible and for the 2-jet process we find the largest corrections for small angles and they reach their minimum for the maximal separation of $\Delta \phi=\pi$.

Another interesting observable, in particular in the context of boosted searches, is the transverse momentum of the diphoton system. This observable is shown in figure 8. Also this observable is only meaningful when having at least one additional jet so we plot this observable only for the 1- and 2-jet process. In both cases the corrections are small for 


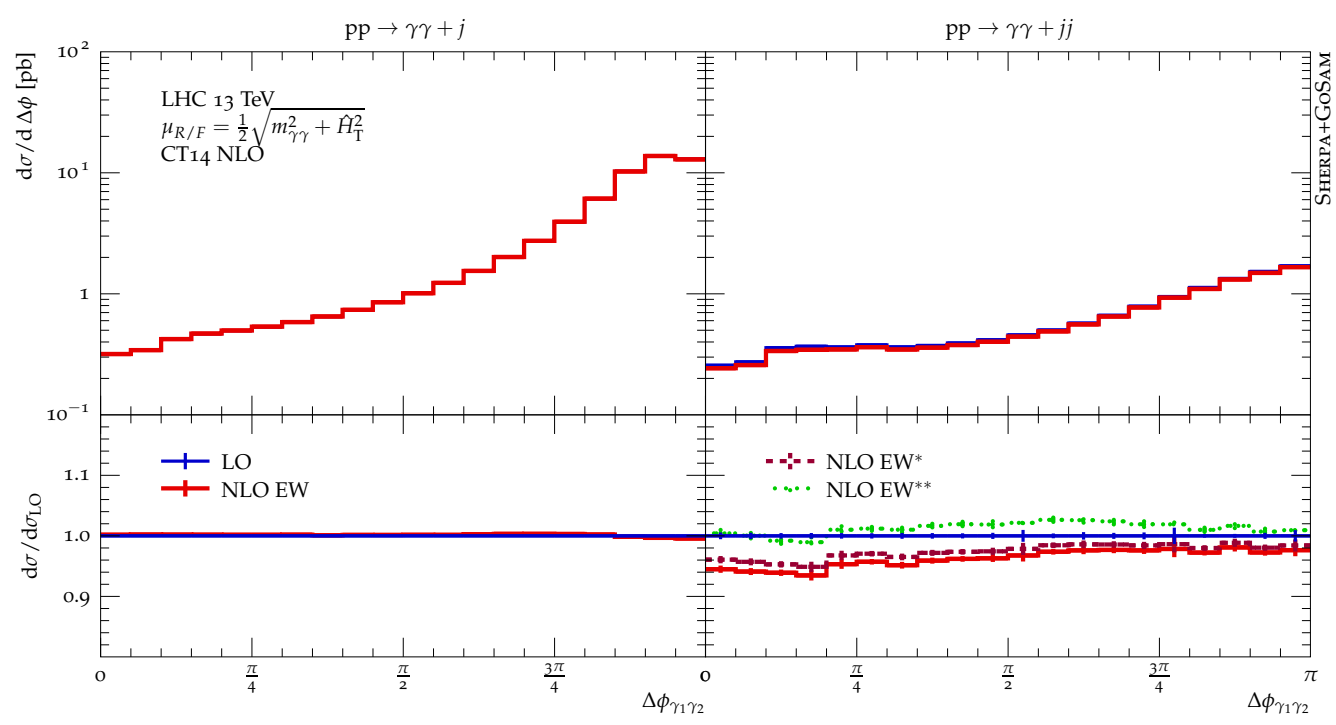

Figure 7. Azimuthal separation of the leading and subleading isolated photon system in $\gamma \gamma+$ 1,2 jets production at $13 \mathrm{TeV}$ at the $\mathrm{LHC}$.

small $p_{\mathrm{T}}$ up to the order of $100 \mathrm{GeV}$ before they become increasingly important leading to $\mathrm{a} \sim 10 \%$ deviation for the 1 -jet process at high transverse momenta of $1 \mathrm{TeV}$. For the 2 -jet process the effects are considerably bigger leading to a $\sim 30 \%$ correction.

Finally, we investigate the $\phi^{*}$ observable in figure 9 . It is defined as $[10,81,82]$

$$
\phi^{*}=\tan \frac{\pi-\Delta \phi_{\gamma \gamma}}{2} \cdot \sin \theta^{*},
$$

where the diphoton opening angle $\theta^{*}$ is defined as

$$
\cos \theta^{*}=\tanh \frac{\left|\Delta y_{\gamma \gamma}\right|}{2} .
$$

It was introduced to provide an alternative measurement to $p_{\mathrm{T}}$, based on well-to-measure particle angles only instead of energy deposits, argued to capture the same physics. It turns out, however, that the 1-jet process does not receive any correction at all, while the 2 -jet process receives moderate, but flat corrections at large $\phi^{*}$. This behaviour is easy to understand since while at least for low transverse momenta $p_{\mathrm{T}}$ and $\phi^{*}$ are very much correlated, this correlation breaks down rather suddenly at $p_{\mathrm{T}} \gtrsim 100 \mathrm{GeV}$ or $\phi^{*} \gtrsim 1$, cf. figure 10. In this region, any value of $\phi^{*}$ receives its largest contribution from $p_{\mathrm{T}}$ regions around $100 \mathrm{GeV}$. Thus, the electroweak corrections can be directly related and vanish for the 1-jet case. Conversely, $\phi^{*}$ is an ineffective probe of high- $p_{\mathrm{T}}$ physics.

Up to this point we have neglected contributions from photon induced processes. As argued above, they are expected to be small due to the smallness of the photon PDF and the fact that these contributions only enter at subleading orders at tree-level. In the following we investigate the actual size of the contributions for two observables where their impact is expected to be largest. They are calculated using the CT14nlo QED [83] set including both an elastic and an inelastic (with an intrinsic photon momentum fraction of 


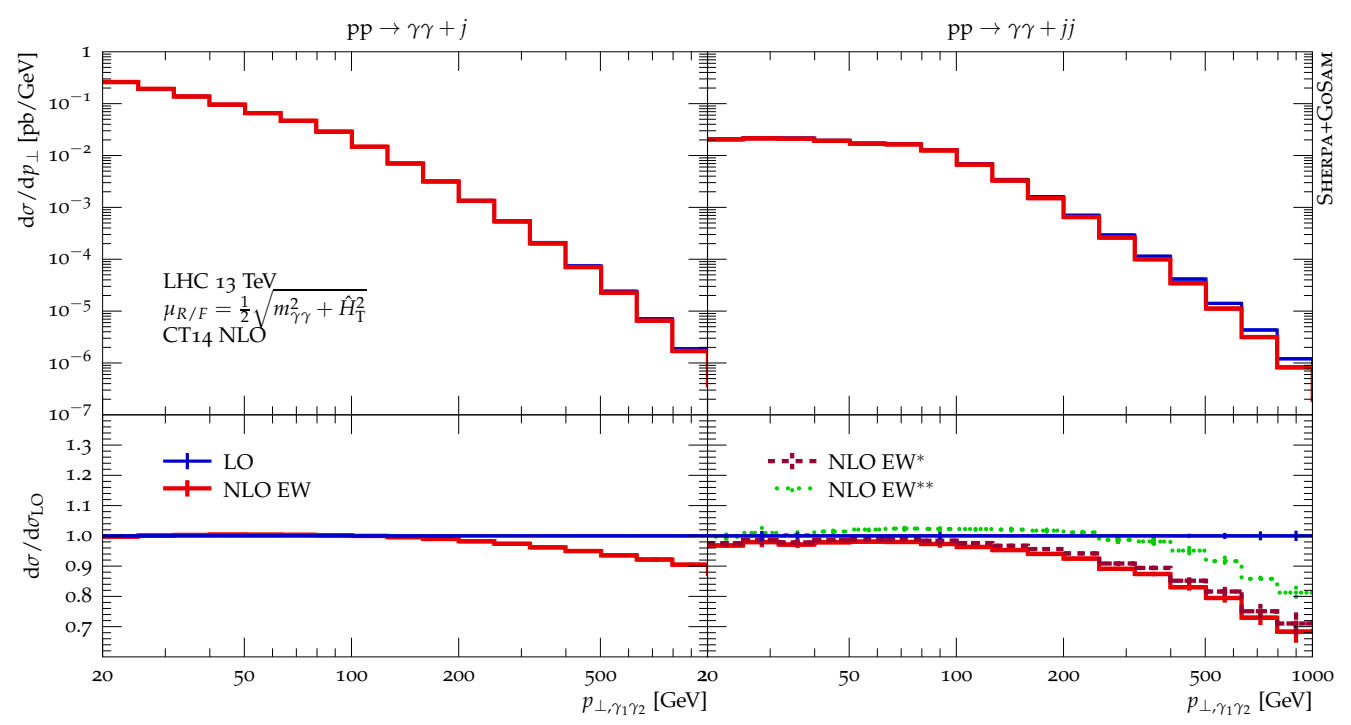

Figure 8. The transverse momentum of the leading and subleading isolated photon system in $\gamma \gamma+1,2$ jets production at $13 \mathrm{TeV}$ at the LHC.

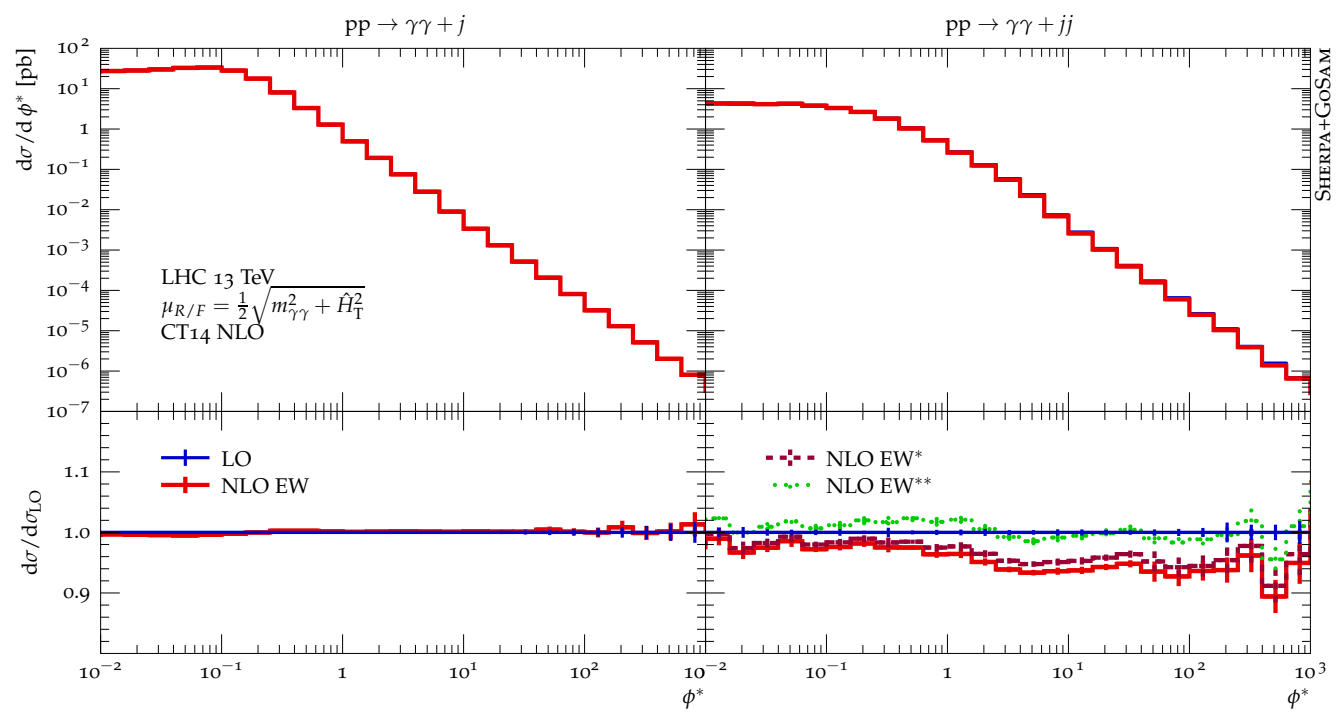

Figure 9. $\phi^{*}$ of the leading and subleading isolated photon system in $\gamma \gamma+1,2$ jets production at $13 \mathrm{TeV}$ at the LHC.

$\left.p_{0}^{\gamma}=0.005\right)$ component, ensuring the quark and gluon PDFs are as close as possible to our nominal set.

Figure 11 details the relative size to the leading tree-level contribution of both the subleading and sub-subleading and the photon-induced contributions for the diphoton invariant mass and the diphoton transverse momentum for both the $\gamma \gamma+1$ jet and $\gamma \gamma+2$ jet processes. For $\gamma \gamma+1$ jet the only subleading contribution is the photon induced one, and it contributes well below $0.5 \%$ throughout the studied range. For $\gamma \gamma+2$ jets the phenomenol- 


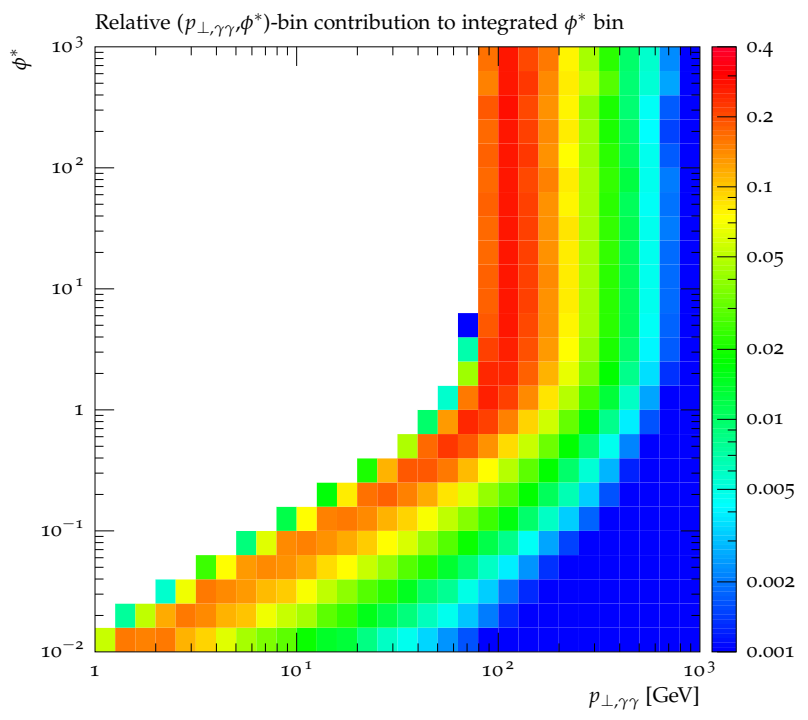

Figure 10. Correlation between the transverse momentum of the diphoton system and $\phi^{*}$ expressed as the fraction each $\left(p_{\mathrm{T}, \gamma \gamma}, \phi^{*}\right)$ bin contributes to the integrated $\phi^{*}$ bin, calculated at LO accuracy (using $q \bar{q} \rightarrow \gamma \gamma g, q g \rightarrow \gamma \gamma q, \bar{q} g \rightarrow \gamma \gamma \bar{q}$ ) at $13 \mathrm{TeV}$ at the LHC. The photon acceptance cuts of eqs. (3.1) and (3.3) are applied, but no jet is required.
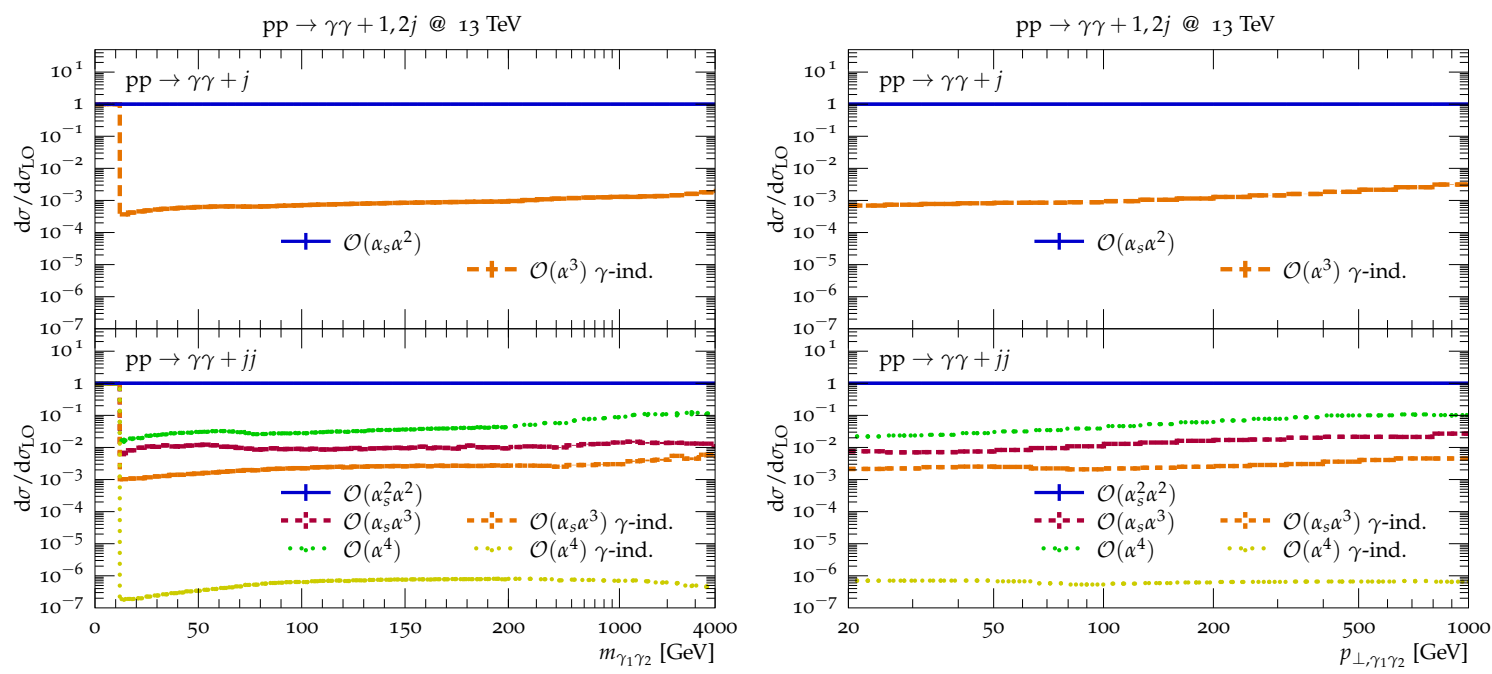

Figure 11. Tree-level contributions from subleading orders and photon initiated processes for the invariant mass and the transverse momentum of the leading and subleading isolated photon system in $\gamma \gamma+1,2$ jets production at $13 \mathrm{TeV}$ at the LHC. Note that on the left plot the $x$-axis is given as a logarithmic scale for values above $200 \mathrm{GeV}$.

ogy is somewhat richer. While, as observed above already, the $\mathcal{O}\left(\alpha^{4}\right)$ sub-subleading contributions are larger than the $\mathcal{O}\left(\alpha_{s} \alpha^{2}\right)$ ones, they increase the cross section at about $10 \%$ and $1 \%$, respectively. The photon-induced processes, on the other hand, again range in the sub-percent region, and are completely negligible. 


\section{Conclusions}

Direct diphoton production channels constitute an important class of background processes for Higgs production in the Standard Model, where the Higgs subsequently decays into a pair of photons, and for many other test of the SM and BSM searches. In this paper we calculated the next-to-leading order electroweak corrections to the production of a photon pair in association with up to two jets. The calculation has been carried out fully automatically using the combination of Sherpa plus GoSam. We restricted ourselves to the leading contributions, i.e the contributions of the orders of $\mathcal{O}\left(\alpha^{2} \alpha_{s}^{i}\right)$ with $i=0,1,2$. For the total cross sections we found corrections between $\sim+1 \%$ and $-3 \%$, depending on the jet multiplicity. As expected the actual impact of the electroweak corrections become mostly effective in the high energy tail of the differential distributions like the transverse momentum, where one easily obtains corrections of the order of $10-20 \%$ for transverse momenta of the order of a few hundred $\mathrm{GeV}$. The inclusion of next-to-leading order electroweak corrections is, therefore, important for a precise prediction of this class of processes.

We also assessed the importance of photon induced processes and find them to be negligible which justifies the usage of pure QCD parton distribution functions. For the 2jet case we also investigated the contributions of subleading and sub-subleading processes (i.e. contributions of $\mathcal{O}\left(\alpha^{3} \alpha_{s}\right)$ and $\mathcal{O}\left(\alpha^{4}\right)$ respectively) at leading order accuracy. We found that the sub-subleading contributions supersede the subleading contributions and lead to corrections that are of similar size as the electroweak corrections to the leading contribution. However, one should note that they are sizeable due to intermediate resonances that can be effectively cut away by applying VBF cuts, in particular by a cut on the invariant mass of the dijet system. We expect that they would lead to a negligible contribution in a VBF type analysis and the $\left(\mathcal{O}\left(\alpha^{2} \alpha_{s}^{2}\right)\right)$ terms would yield the dominant contribution.

\section{Acknowledgments}

We would like to thank Jonas Lindert and Stefano Pozzorini for comparing results. N.G. was supported by the Swiss National Science Foundation under contract PZ00P2_154829. M.S. was supported by PITN-GA-2012-315877 (MCnet) and the ERC Advanced Grant MC@NNLO (340983). FT was supported in part by MIUR under Project No. 2015P5SBHT and by the INFN research initiative ENP.

Open Access. This article is distributed under the terms of the Creative Commons Attribution License (CC-BY 4.0), which permits any use, distribution and reproduction in any medium, provided the original author(s) and source are credited.

\section{References}

[1] ATLAS collaboration, Observation of a new particle in the search for the Standard Model Higgs boson with the ATLAS detector at the LHC, Phys. Lett. B 716 (2012) 1 [arXiv:1207.7214] [INSPIRE]. 
[2] CMS collaboration, Observation of a new boson at a mass of $125 \mathrm{GeV}$ with the CMS experiment at the LHC, Phys. Lett. B 716 (2012) 30 [arXiv:1207.7235] [INSPIRE].

[3] ATLAS collaboration, Measurement of Higgs boson production in the diphoton decay channel in pp collisions at center-of-mass energies of 7 and 8 TeV with the ATLAS detector, Phys. Rev. D 90 (2014) 112015 [arXiv: 1408.7084] [INSPIRE].

[4] ATLAS collaboration, Measurements of fiducial and differential cross sections for Higgs boson production in the diphoton decay channel at $\sqrt{s}=8$ TeV with ATLAS, JHEP 09 (2014) 112 [arXiv:1407.4222] [INSPIRE].

[5] CMS collaboration, Measurement of differential fiducial cross sections for Higgs boson production in the diphoton decay channel in pp collisions at $\sqrt{s}=13 \mathrm{TeV}$, CMS-PAS-HIG-17-015 (2017).

[6] CMS collaboration, Updated measurements of Higgs boson production in the diphoton decay channel at $\sqrt{s}=13$ TeV in pp collisions at CMS, CMS-PAS-HIG-16-020 (2016).

[7] CMS collaboration, Measurement of differential cross sections for Higgs boson production in the diphoton decay channel in pp collisions at $\sqrt{s}=8$ TeV, Eur. Phys. J. C 76 (2016) 13 [arXiv: 1508.07819] [INSPIRE].

[8] ATLAS collaboration, Measurement of the isolated di-photon cross-section in pp collisions at $\sqrt{s}=7$ TeV with the ATLAS detector, Phys. Rev. D 85 (2012) 012003 [arXiv:1107.0581] [INSPIRE].

[9] ATLAS collaboration, Measurement of isolated-photon pair production in pp collisions at $\sqrt{s}=7 \mathrm{TeV}$ with the ATLAS detector, JHEP 01 (2013) 086 [arXiv:1211.1913] [INSPIRE].

[10] ATLAS collaboration, Measurements of integrated and differential cross sections for isolated photon pair production in pp collisions at $\sqrt{s}=8 \mathrm{TeV}$ with the ATLAS detector, Phys. Rev. D 95 (2017) 112005 [arXiv:1704.03839] [INSPIRE].

[11] CMS collaboration, Measurement of the Production Cross Section for Pairs of Isolated Photons in pp collisions at $\sqrt{s}=7$ TeV, JHEP 01 (2012) 133 [arXiv:1110.6461] [INSPIRE].

[12] CMS collaboration, Measurement of differential cross sections for the production of a pair of isolated photons in pp collisions at $\sqrt{s}=7$ TeV, Eur. Phys. J. C 74 (2014) 3129 [arXiv:1405.7225] [INSPIRE].

[13] ATLAS collaboration, Search for scalar diphoton resonances with $15.4 \mathrm{fb}^{-1}$ of data collected at $\sqrt{s}=13$ TeV in 2015 and 2016 with the ATLAS detector, ATLAS-CONF-2016-059 (2016).

[14] ATLAS collaboration, Search for resonances in diphoton events at $\sqrt{s}=13$ TeV with the ATLAS detector, JHEP 09 (2016) 001 [arXiv: 1606.03833] [INSPIRE].

[15] ATLAS collaboration, Search for nonpointing and delayed photons in the diphoton and missing transverse momentum final state in $8 \mathrm{TeV}$ pp collisions at the LHC using the ATLAS detector, Phys. Rev. D 90 (2014) 112005 [arXiv: 1409.5542] [INSPIRE].

[16] ATLAS collaboration, Search for Scalar Diphoton Resonances in the Mass Range 65-600 GeV with the ATLAS Detector in pp Collision Data at $\sqrt{s}=8$ TeV, Phys. Rev. Lett. 113 (2014) 171801 [arXiv: 1407.6583] [INSPIRE].

[17] CMS collaboration, Search for high-mass diphoton resonances in proton-proton collisions at 13 TeV and combination with 8 TeV search, Phys. Lett. B 767 (2017) 147 [arXiv: 1609.02507] [INSPIRE]. 
[18] CMS collaboration, Search for new physics in high mass diphoton events in $3.3 \mathrm{fb}^{-1}$ of proton-proton collisions at $\sqrt{s}=13 \mathrm{TeV}$ and combined interpretation of searches at $8 \mathrm{TeV}$ and $13 \mathrm{TeV}$, CMS-PAS-EXO-16-018 (2016).

[19] CMS collaboration, Search for new physics in high mass diphoton events in proton-proton collisions at $\sqrt{s}=13 \mathrm{TeV}$, CMS-PAS-EXO-15-004 (2015).

[20] CMS collaboration, Search for diphoton resonances in the mass range from 150 to $850 \mathrm{GeV}$ in pp collisions at $\sqrt{s}=8 \mathrm{TeV}$, Phys. Lett. B 750 (2015) 494 [arXiv:1506. 02301] [InSPIRE].

[21] P. Aurenche, A. Douiri, R. Baier, M. Fontannaz and D. Schiff, Large $p_{T}$ Double Photon Production in Hadronic Collisions: Beyond Leading Logarithm QCD Calculation, Z. Phys. C 29 (1985) 459 [INSPIRE].

[22] B. Bailey, J.F. Owens and J. Ohnemus, An Order $\alpha_{s}$ Monte Carlo calculation of hadronic double photon production, Phys. Rev. D 46 (1992) 2018 [InSPIRE].

[23] T. Binoth, J.P. Guillet, E. Pilon and M. Werlen, A full next-to-leading order study of direct photon pair production in hadronic collisions, Eur. Phys. J. C 16 (2000) 311 [hep-ph/9911340] [INSPIRE].

[24] Z. Bern, L.J. Dixon and C. Schmidt, Isolating a light Higgs boson from the diphoton background at the CERN LHC, Phys. Rev. D 66 (2002) 074018 [hep-ph/0206194] [INSPIRE].

[25] J.M. Campbell, R.K. Ellis and C. Williams, Vector boson pair production at the LHC, JHEP 07 (2011) 018 [arXiv: 1105.0020] [INSPIRE].

[26] K. Arnold et al., VBFNLO: A Parton level Monte Carlo for processes with electroweak bosons, Comput. Phys. Commun. 180 (2009) 1661 [arXiv:0811.4559] [InSPIRE].

[27] L. D'Errico and P. Richardson, Next-to-Leading-Order Monte Carlo Simulation of Diphoton Production in Hadronic Collisions, JHEP 02 (2012) 130 [arXiv:1106.3939] [INSPIRE].

[28] S. Hoeche, S. Schumann and F. Siegert, Hard photon production and matrix-element parton-shower merging, Phys. Rev. D 81 (2010) 034026 [arXiv:0912.3501] [INSPIRE].

[29] C. Balázs, E.L. Berger, P.M. Nadolsky and C.P. Yuan, All-orders resummation for diphoton production at hadron colliders, Phys. Lett. B 637 (2006) 235 [hep-ph/0603037] [INSPIRE].

[30] C. Balázs, P.M. Nadolsky, C. Schmidt and C.P. Yuan, Diphoton background to Higgs boson production at the LHC with soft gluon effects, Phys. Lett. B 489 (2000) 157 [hep-ph/9905551] [INSPIRE].

[31] L. Cieri, F. Coradeschi and D. de Florian, Diphoton production at hadron colliders: transverse-momentum resummation at next-to-next-to-leading logarithmic accuracy, JHEP 06 (2015) 185 [arXiv: 1505.03162] [INSPIRE].

[32] S. Catani, L. Cieri, D. de Florian, G. Ferrera and M. Grazzini, Diphoton production at hadron colliders: a fully-differential QCD calculation at NNLO, Phys. Rev. Lett. 108 (2012) 072001 [arXiv: 1110.2375 ] [INSPIRE].

[33] J.M. Campbell, R.K. Ellis, Y. Li and C. Williams, Predictions for diphoton production at the LHC through NNLO in QCD, JHEP 07 (2016) 148 [arXiv: 1603.02663] [INSPIRE].

[34] A. Bierweiler, T. Kasprzik and J.H. Kühn, Vector-boson pair production at the LHC to $\mathcal{O}\left(\alpha^{3}\right)$ accuracy, JHEP 12 (2013) 071 [arXiv: 1305. 5402] [INSPIRE]. 
[35] V. Del Duca, F. Maltoni, Z. Nagy and Z. Trócsányi, QCD radiative corrections to prompt diphoton production in association with a jet at hadron colliders, JHEP 04 (2003) 059 [hep-ph/0303012] [INSPIRE].

[36] T. Gehrmann, N. Greiner and G. Heinrich, Photon isolation effects at $N L O$ in $\gamma \gamma+$ jet final states in hadronic collisions, JHEP 06 (2013) 058 [Erratum ibid. 1406 (2014) 076] [arXiv: 1303.0824] [INSPIRE].

[37] T. Gehrmann, N. Greiner and G. Heinrich, Precise QCD predictions for the production of a photon pair in association with two jets, Phys. Rev. Lett. 111 (2013) 222002 [arXiv: 1308.3660] [INSPIRE].

[38] S. Badger, A. Guffanti and V. Yundin, Next-to-leading order QCD corrections to di-photon production in association with up to three jets at the Large Hadron Collider, JHEP 03 (2014) 122 [arXiv: 1312.5927] [INSPIRE].

[39] Z. Bern et al., Next-to-leading order $\gamma \gamma+2$-jet production at the LHC, Phys. Rev. D 90 (2014) 054004 [arXiv: 1402.4127] [INSPIRE].

[40] G. Cullen et al., Automated One-Loop Calculations with GoSam, Eur. Phys. J. C 72 (2012) 1889 [arXiv: 1111.2034] [INSPIRE].

[41] G. Cullen et al., GoSam-2.0: a tool for automated one-loop calculations within the Standard Model and beyond, Eur. Phys. J. C 74 (2014) 3001 [arXiv: 1404.7096] [INSPIRE].

[42] T. Gleisberg et al., Event generation with SHERPA 1.1, JHEP 02 (2009) 007 [arXiv:0811.4622] [INSPIRE].

[43] P. Nogueira, Automatic Feynman graph generation, J. Comput. Phys. 105 (1993) 279.

[44] J.A.M. Vermaseren, New features of FORM, math-ph/0010025 [INSPIRE].

[45] J. Kuipers, T. Ueda, J.A.M. Vermaseren and J. Vollinga, FORM version 4.0, Comput. Phys. Commun. 184 (2013) 1453 [arXiv:1203.6543] [INSPIRE].

[46] G. Cullen, M. Koch-Janusz and T. Reiter, Spinney: A Form Library for Helicity Spinors, Comput. Phys. Commun. 182 (2011) 2368 [arXiv:1008.0803] [InSPIRE].

[47] T. Peraro, Ninja: Automated Integrand Reduction via Laurent Expansion for One-Loop Amplitudes, Comput. Phys. Commun. 185 (2014) 2771 [arXiv:1403.1229] [INSPIRE].

[48] P. Mastrolia, E. Mirabella and T. Peraro, Integrand reduction of one-loop scattering amplitudes through Laurent series expansion, JHEP 06 (2012) 095 [Erratum ibid. 1211 (2012) 128] [arXiv: 1203.0291] [INSPIRE].

[49] H. van Deurzen, G. Luisoni, P. Mastrolia, E. Mirabella, G. Ossola and T. Peraro, Multi-leg One-loop Massive Amplitudes from Integrand Reduction via Laurent Expansion, JHEP 03 (2014) 115 [arXiv:1312.6678] [INSPIRE].

[50] G. Ossola, C.G. Papadopoulos and R. Pittau, Reducing full one-loop amplitudes to scalar integrals at the integrand level, Nucl. Phys. B 763 (2007) 147 [hep-ph/0609007] [InSPIRE].

[51] P. Mastrolia, G. Ossola, C.G. Papadopoulos and R. Pittau, Optimizing the Reduction of One-Loop Amplitudes, JHEP 06 (2008) 030 [arXiv: 0803.3964] [INSPIRE].

[52] G. Ossola, C.G. Papadopoulos and R. Pittau, On the Rational Terms of the one-loop amplitudes, JHEP 05 (2008) 004 [arXiv:0802.1876] [inSPIRE]. 
[53] P. Mastrolia, G. Ossola, T. Reiter and F. Tramontano, Scattering AMplitudes from Unitarity-based Reduction Algorithm at the Integrand-level, JHEP 08 (2010) 080 [arXiv: 1006.0710] [INSPIRE].

[54] G. Heinrich, G. Ossola, T. Reiter and F. Tramontano, Tensorial Reconstruction at the Integrand Level, JHEP 10 (2010) 105 [arXiv: 1008.2441] [INSPIRE].

[55] T. Binoth, J.P. Guillet, G. Heinrich, E. Pilon and T. Reiter, Golem95: A Numerical program to calculate one-loop tensor integrals with up to six external legs, Comput. Phys. Commun. 180 (2009) 2317 [arXiv:0810.0992] [INSPIRE].

[56] G. Cullen et al., Golem95C: A library for one-loop integrals with complex masses, Comput. Phys. Commun. 182 (2011) 2276 [arXiv:1101.5595] [INSPIRE].

[57] J.P. Guillet, G. Heinrich and J.F. von Soden-Fraunhofen, Tools for NLO automation: extension of the golem95C integral library, Comput. Phys. Commun. 185 (2014) 1828 [arXiv: 1312.3887] [INSPIRE].

[58] A. van Hameren, OneLOop: For the evaluation of one-loop scalar functions, Comput. Phys. Commun. 182 (2011) 2427 [arXiv: 1007.4716] [INSPIRE].

[59] M. Chiesa, N. Greiner and F. Tramontano, Automation of electroweak corrections for LHC processes, J. Phys. G 43 (2016) 013002 [arXiv:1507.08579] [InSPIRE].

[60] A. Denner, Techniques for calculation of electroweak radiative corrections at the one loop level and results for W physics at LEP-200, Fortsch. Phys. 41 (1993) 307 [arXiv:0709.1075] [INSPIRE].

[61] A. Denner, S. Dittmaier, M. Roth and D. Wackeroth, Predictions for all processes $e^{+} e^{-} \rightarrow 4$ fermions + gamma, Nucl. Phys. B 560 (1999) 33 [hep-ph/9904472] [InSPIRE].

[62] A. Denner, S. Dittmaier, M. Roth and L.H. Wieders, Electroweak corrections to charged-current $e^{+} e^{-} \rightarrow 4$ fermion processes: Technical details and further results, Nucl. Phys. B 724 (2005) 247 [Erratum ibid. B 854 (2012) 504] [hep-ph/0505042] [INSPIRE].

[63] A. Denner and S. Dittmaier, The Complex-mass scheme for perturbative calculations with unstable particles, Nucl. Phys. Proc. Suppl. 160 (2006) 22 [hep-ph/0605312] [INSPIRE].

[64] F. Krauss, R. Kuhn and G. Soff, AMEGIC++ 1.0: A Matrix element generator in $\mathrm{C}++$, JHEP 02 (2002) 044 [hep-ph/0109036] [INSPIRE].

[65] S. Catani and M.H. Seymour, A General algorithm for calculating jet cross-sections in NLO QCD, Nucl. Phys. B 485 (1997) 291 [Erratum ibid. B 510 (1998) 503] [hep-ph/9605323] [INSPIRE].

[66] S. Dittmaier, A General approach to photon radiation off fermions, Nucl. Phys. B 565 (2000) 69 [hep-ph/9904440] [INSPIRE].

[67] T. Gleisberg and F. Krauss, Automating dipole subtraction for QCD NLO calculations, Eur. Phys. J. C 53 (2008) 501 [arXiv:0709.2881] [INSPIRE].

[68] M. Schönherr, An automated subtraction of NLO EW infrared divergences, in preparation.

[69] S. Kallweit, J.M. Lindert, P. Maierhöfer, S. Pozzorini and M. Schönherr, NLO electroweak automation and precise predictions for W+multijet production at the LHC, JHEP 04 (2015) 012 [arXiv: 1412.5157] [INSPIRE]. 
[70] S. Kallweit, J.M. Lindert, P. Maierhöfer, S. Pozzorini and M. Schönherr, $N L O Q C D+E W$ predictions for $V+$ jets including off-shell vector-boson decays and multijet merging, JHEP 04 (2016) 021 [arXiv: 1511.08692] [INSPIRE].

[71] S. Kallweit, J.M. Lindert, S. Pozzorini and M. Schönherr, NLO QCD+EW predictions for $2 \ell 2 \nu$ diboson signatures at the $L H C$, arXiv: 1705.00598 [INSPIRE].

[72] T. Binoth et al., A Proposal for a standard interface between Monte Carlo tools and one-loop programs, Comput. Phys. Commun. 181 (2010) 1612 [arXiv:1001.1307] [INSPIRE].

[73] S. Alioli et al., Update of the Binoth Les Houches Accord for a standard interface between Monte Carlo tools and one-loop programs, Comput. Phys. Commun. 185 (2014) 560 [arXiv: 1308.3462] [INSPIRE].

[74] S. Dulat et al., New parton distribution functions from a global analysis of quantum chromodynamics, Phys. Rev. D 93 (2016) 033006 [arXiv: 1506.07443] [INSPIRE].

[75] A. Buckley et al., LHAPDF6: parton density access in the LHC precision era, Eur. Phys. J. C 75 (2015) 132 [arXiv: 1412.7420] [INSPIRE].

[76] S. Frixione, Isolated photons in perturbative QCD, Phys. Lett. B 429 (1998) 369 [hep-ph/9801442] [INSPIRE].

[77] E.W.N. Glover and A.G. Morgan, Measuring the photon fragmentation function at LEP, Z. Phys. C 62 (1994) 311 [INSPIRE].

[78] M. Cacciari, G.P. Salam and G. Soyez, The Anti-k(t) jet clustering algorithm, JHEP 04 (2008) 063 [arXiv: 0802.1189] [INSPIRE].

[79] M. Cacciari, G.P. Salam and G. Soyez, FastJet User Manual, Eur. Phys. J. C 72 (2012) 1896 [arXiv: 1111.6097] [INSPIRE].

[80] M. Cacciari and G.P. Salam, Dispelling the $N^{3}$ myth for the $k_{t}$ jet-finder, Phys. Lett. B 641 (2006) 57 [hep-ph/0512210] [INSPIRE].

[81] M. Vesterinen and T.R. Wyatt, A Novel Technique for Studying the $Z$ Boson Transverse Momentum Distribution at Hadron Colliders, Nucl. Instrum. Meth. A 602 (2009) 432 [arXiv: 0807 .4956] [INSPIRE].

[82] A. Banfi, S. Redford, M. Vesterinen, P. Waller and T.R. Wyatt, Optimisation of variables for studying dilepton transverse momentum distributions at hadron colliders, Eur. Phys. J. C 71 (2011) 1600 [arXiv: 1009.1580] [inSPIRE].

[83] C. Schmidt, J. Pumplin, D. Stump and C.P. Yuan, CT14QED parton distribution functions from isolated photon production in deep inelastic scattering, Phys. Rev. D 93 (2016) 114015 [arXiv: 1509.02905] [INSPIRE]. 